\title{
Riesgo de apnea obstructiva del sueño en pacientes quirúrgicos de una institución de salud, Pereira, 2019
}

\author{
Daniela Benjumea Oyola', Eliana Vanessa Arango², Angélica María Blanco ${ }^{3}$, \\ Germán Acevedo Osorio ${ }^{4}$, Janeth Cecilia Alvarado Luna ${ }^{4}$
}

RESUMEN Introducción: los pacientes con diagnóstico de síndrome de Apnea-Hipopnea Obstructiva del Sueño (SAHOS), pueden ser llevados de forma segura a cualquier procedimiento quirúrgico, incluso de manera ambulatoria, si tienen control adecuado de sus otras comorbilidades La sedación debe ser hecha por un anestesiólogo, quien, además, debe vigilar al paciente y disponer del equipo adecuado para atender complicaciones respiratorias emergentes. Es importante que desde la valoración pre anestésica se identifique a los pacientes con riesgo de SAHOS, para trazar un plan anestésico que disminuya las complicaciones a nivel respiratorio y del manejo de la vía área.

Objetivo: establecer el riesgo de apnea obstructiva del sueño en un grupo de pacientes quirúrgicos, en una institución de salud en el municipio de Pereira, 2019.

Materiales y métodos: tipo de estudio descriptivo transversal prospectivo. La población está conformada por pacientes mayores de 18 años, programados para cirugías electivas, en quienes se utilizará anestesia general o sedación, sin diagnóstico previo de apnea obstructiva del sueño, en óptimas condiciones para responder verbalmente el cuestionario, y si desean participar en el estudio. La información se recolectará mediante el instrumento Stop-Bang, el cual tiene como fin, proporcionar la indagación necesaria para el logro de los objetivos de la investigación, y determinar la severidad del SAHOS. La información será analizada en SPSS versión 23

PALABRAS Complicaciones Intra Operatorias;

CLAVE $\quad$ Periodo Peri Operatorio;

Procedimientos Quirúrgicos Operativos.

1 Instrumentación Quirúrgica, IV Semestre, Fundación Universitaria Del Área Andina, dbenjumea6@estudiantes.areandina.edu.co

2 Instrumentación Quirúrgica, IV Semestre, Fundación Universitaria Del Área Andina, earango12@estudiantes.areandina.edu.co

3 Asesora temática, Terapia Respiratoria, Fundación Universitaria Del Área Andina

4 Asesor, Instrumentación Quirúrgica, Fundación Universitaria Del Área Andina 


\section{Risk of obstructive sleep apnea in surgical patients at a health institution, Pereira, 2019}

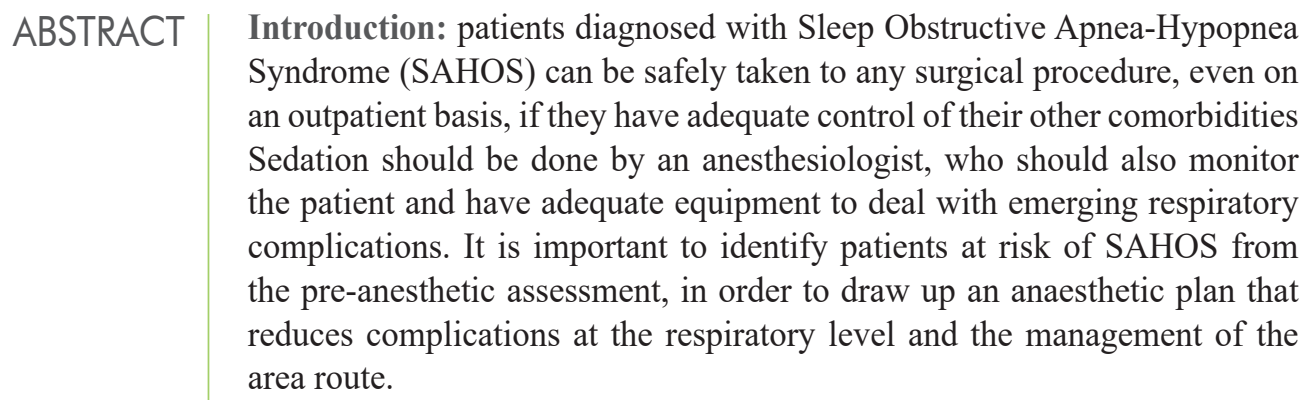

Objective: to establish the risk of obstructive sleep apnea in a group of surgical patients at a health institution in the municipality of Pereira, 2019.

Materials and methods: type of prospective cross-sectional descriptive study. The population consists of patients over 18 years of age, scheduled for elective surgery, in whom general anesthesia or sedation will be used, without prior diagnosis of obstructive sleep apnea, in optimal conditions to respond verbally to the questionnaire, and if they wish to participate in the study. The information will be collected through the Stop-Bang instrument, which aims to provide the necessary research for the achievement of the research objectives, and to determine the severity of the SAHOS. The information will be analyzed in SPSS version 23.

Obstructive Sleep Apnea;

Intra-Operative Complications;

Peri-Operative Period;

Operative Surgical Procedures. 


\section{INTRODUCCIÓN}

El incremento de la apnea obstructiva del sueño (SAHOS), es un factor poblacional importante que ha tomado auge en la última década y se cree que aumentará en los próximos años, magnificándolo como un problema de salud pública y convirtiéndose en un detonante de patologías y riesgos peri operatorios, que se desencadenan a mayor escala cuando el paciente presenta alteraciones anatómicas de la vía aérea superior, encontrándose mayor dificultad en el proceso de intubación y exigiendo utilizar una técnica especializada. Con estos pacientes se debe tener un estricto cuidado en la monitorización de la vía aérea superior, medicación y control de signos vitales(1).

La SAHOS presenta una alta prevalencia entre la población general (4 y 2\% en hombres y mujeres respectivamente), y está relacionada con un gran número de comorbilidades: enfermedad cardiovascular, arritmia, infarto, obesidad, síndrome metabólico, resistencia a la insulina, depresión, e incluso no tratada puede derivar en hipertensión arterial no controlada; falla cardiaca y muerte súbita. Se sabe que la vía aérea de estos pacientes conlleva una serie de particularidades con relación a la ventilación con mascarilla, la intubación traqueal y la ventilación post-extubación. El incremento de la tendencia al colapso de la vía aérea y en la sensibilidad a los depresores del SNC, añaden complejidad al manejo patológico; así mismo, es habitual que muchos pacientes con SAHOS no estén formalmente diagnosticados (hasta un $80-90 \%)$. Por esta causa, un diagnóstico temprano podría cambiar resultados en el peri operatorio (1).

El riesgo peri operativo de los pacientes con SAHOS ha sido subestimado por el equipo quirúrgico. Con relación a la vía aérea se establece como uno de los factores que podría suponer un incremento en la dificultad para su manejo. En concreto, para la ventilación con mascarilla facial, el manejo peri operatorio de estos pacientes requiere vigilancia de las comorbilidades asociadas, la preparación para una posible dificultad de la vía aérea, la profilaxis en la aspiración pulmonar, evitándose factores que contribuyen a la depresión respiratoria postoperatoria, siendo imprescindible establecer un alto nivel de sospecha previo a la cirugía, y un estricto control de la vía aérea durante todo el proceso (1).

En el transcurso peri operatorio de pacientes con SAHOS, es indispensable verificar el grado de severidad de la patología con el estudio polisomnográfico y la medicación que está utilizando el paciente, debido a que los tratamientos con benzodiacepinas u opioides suelen reducir el tono muscular y la luz de la vía aérea superior; deprimen el sistema nervioso central, producen alteraciones en la conducta cardiaca e hipoventilación, aumentando así el riesgo de complicaciones pulmonares (2).

El acto anestésico debe ser estricto y cuidadoso, dependiendo de factores físicos y patológicos del paciente; este puede verse afectado cuando el anestésico suministrado es de larga duración, llevando a un retraso en el estado de consciencia del paciente 136 y la recuperación de su vía aérea; la anestesia general no permite tener un individuo 
alerta e incrementa el umbral del dolor. El quirófano debe contar con un equipo especializado en el momento de suministrar la anestesia, de lo contrario favorecerá alteraciones estructurales que condicionarían una vía aérea difícil.(3).

Los pacientes sometidos a cirugía de vía aérea como cirugía de avance mandibular, suspensión de la lengua, adenoamigalectomia, cirugía nasal, avance genio gloso, traqueotomía, turbinectomía entre otras, se exponen a complicaciones como son: hemorragia, infecciones, disfunción articular, dehiscencia, iatrogenia de paquetes vasculares importantes y de estructuras anatómicas. Con lo anterior, se incrementan los factores de riesgo cuando el paciente presenta patologías de base tales como la obesidad, alteraciones severas craneofaciales o pacientes con manejo de sonda nasogástrica, puesto que se presentará una intubación y un postoperatorio más complejo (4).

Respecto a las consideraciones del impacto postoperatorio, se evidencian en las 72 primeras horas inmediatas; el acto más importante es la extubación que no debe hacerse de manera inmediata ya que en este momento el paciente se verá expuesto a hipercapnia, hipertensión arterial pulmonar, sistémica, aumento de la presión intratorácica, aumento en la carga en los ventrículos, bradicardia, arritmia, alteraciones craneofaciales, aumento de la presión arterial, sialoadenitis, trastorno salivales, deshidratación, edemas, hemorragia gastrointestinal retardada, hipoestaesia del labio inferior, taponamientos nasales que generan un mayor esfuerzo respiratorio e incrementan exponencialmente la posibilidad el colapso aéreo; una posición e inclinación incorrecta llevará a el paciente a una obstrucción de la vía aérea superior(5)

Se estima que un mal diagnóstico acompañado de complicaciones en el procedimiento peri operatorio podría conllevar a una prolongación de la estancia hospitalaria, trayendo como consecuencia sobrecarga en el servicio médico, modificación en los horarios y rutinas del personal, alteración en los protocolos y políticas de la institución, como lo es la distribución de camas, distorsión organizativa, demora en la tramitación de pruebas diagnósticas, retraso en los horarios de visita, aumento en la posibilidad de adquirir otra patología del sitio hospitalario; concluyendo que estos factores enmarcados anteriormente traen como consecuencia un aumento en los activos de la institución, recursos que podrían ser empleados en otra área para mejorar la atención (6).

Por los motivos expuestos, se plantea la siguiente pregunta ¿Cuál es el riesgo de la apnea obstructiva de sueño en pacientes quirúrgicos en una institución de salud, Pereira, 2019?

\section{METODOLOGÍA}

Tipo de estudio: estudio descriptivo transversal prospectivo. 


\section{Población y muestra}

Criterios de inclusión: pacientes mayores de 18 años, programados para cirugías electivas en quienes se utilizará anestesia general o sedación, sin diagnóstico previo de apnea obstructiva del sueño, en óptimas condiciones para responder verbalmente el cuestionario y si desean participar en el estudio.

Criterios de exclusión: pacientes con trastornos mentales, que les ocasione dificultad la conciliación del sueño, tales como depresión, insomnio o psicosis; pacientes que por algún motivo durante los procedimientos diagnósticos invasivos hayan rechazado la sedación; pacientes con enfermedades neurológicas, con trastornos de sueño diagnosticados, enfermedad terminal o estar inmovilizado, pacientes en estado de urgencia.

Recolección de información: se recolectará mediante el instrumento Stop-Bang, el cual tiene como objetivo proporcionar la información necesaria para el logro de los propósitos de la investigación y determinar la severidad del SAHOS. El cuestionario Alfa de Cronbach de 0.8 para apnea del sueño severa; la información será tabulada en Excel 2019.

Análisis de Información: la información será analizada en SPSS versión 23; las variables cualitativas se describirán mediante tablas de frecuencias (absolutas, relativas); para las variables cuantitativas se utilizarán medidas de tendencia central y medidas de dispersión; para el análisis bivariado se utilizará la prueba Chi cuadrado de Pearson; para el análisis cuantitativo se aplicarán pruebas paramétricas y no paramétricas según corresponda su distribución.

Componente Bioético: de acuerdo de la resolución 008430 e 1993 y al Comité de Investigación del Programa de Instrumentación Quirúrgica, el estudio se considera sin riesgo, por lo cual se garantizará la confidencialidad de la información y participación voluntaria en el mismo, previa firma del consentimiento informado.

\section{RESULTADOS ESPERADOS}

- Se espera establecer el grado de riesgo que presentan los pacientes con apnea obstructiva de sueño relacionada con las variables sociodemográfica y clínicas, buscando asociaciones estadísticas, con el fin de informar a la comunidad hospitalaria la importancia de la detección de dicho trastorno, previo a la aplicación de la anestesia, y presentar el cuestionario STOPBANG como una alternativa en el preoperatorio.

Los resultados serán divulgados en eventos de investigación del área de interés y 
1. Ana AF, Patricia HP. Manejo perioperatorio del paciente con síndrome de apneahipopnea obstructiva del sueño ( SAHOS ). 2017;65:81-5.

2. Vega-osorio SPPA. Factores de riesgo y asociados al síndrome de apnea-hipopnea obstructiva del sueño ( SAHOS ). 2017;65:21-4.

3. Baptista PM. Cirugía como tratamiento de la apnea obstructiva del sueño Surgery for obstructive sleep apnea. 2007;30.

4. Respiratori G. Manejo perioperatorio de la apnea-hipopnea del sueño ( SAHS ). 2015;

5. Covarrubias-gómez A, Guevara-lópez U, Haro-valencia R, Alvarado-suárez M. Artemisa del sueño y su importancia en la medicina perioperatoria. 2006;371-80.

6. Dixon SE, Haas SA, Klopp A, Carlson J. ORIGINAL ARTICLES A Quality Improvement Project: Using the STOP-BANG Tool in a Military Population to Improve Equity in Preoperative Screening. J PeriAnesthesia Nurs [Internet]. Elsevier; 2016;31(5):371-80. Available from: http://dx.doi.org/10.1016/j. jopan.2014.12.002 